\title{
Érasme, pédagogue du bonheur, dans les Colloques
}

\author{
BRENDA DUNN-LARDEAU \\ Université du Québec à Montréal
}

In 1518, Erasmus published The Colloquies (Colloquia), a lively Latin conversation primer, which acquainted European youths with a variety of subjects, including that of happiness. This article examines several of The Colloquies, but most notably the Senile Colloquium, to assess Erasmus' response to the question of the changing interrelationship of the two felicities - celestial and earthly — during the Renaissance. He asserts that personal happiness is no longer scorned upon as in the Middle Ages provided that honestas and voluptas walk hand in hand and philautia steer away for the sake of balance between individual and collective happiness. In addition, The Colloquies, which include pleasure seekers from all walks of life, exposes young readers to a moral discourse, wherein pagan and Christian models mingle, while avoiding the moralizing tone of earlier medieval morality texts. As for the Senile Colloquium, published in 1524 (while Erasmus wrote his Diatribè sive Collatio de libero arbitrio), it distinguishes true and false happiness and adapts the classical models of the conduct of life (voluptuous, active, contemplative) to a Christian society. Erasmus considers, as did Aristotle, that mankind is responsible for its happiness but makes the philosophia Christi the best way to achieve it.

\section{De la Félicité dans les Colloques}

$\mathrm{C}$ ontrairement au discours médiéval qui mettait l'emphase sur la béatitude éternelle, celui de la Renaissance affiche sa volonté de concilier les deux félicités, céleste et terrestre ${ }^{1}$. L'objet de cet article est de mettre au jour cette volonté typiquement renaissante dans les Colloques d'Érasme où il accorde une place importante à la félicité humaine. En effet, dans bon nombre d'entre eux, Érasme a mis en scène une variété de modèles de félicité qui touchent plusieurs catégories sociales. Plus particulièrement, Le Colloque des vieillards ou le Coche (Senile Colloquium) fournira l'occasion d'examiner la pédagogie sur le bonheur (ou la rhétorique du « joyeux entretien » selon l'expression d'Érasme), qui se voit mise au service des idées sur la félicité ${ }^{2}$.

Renaissance and Reformation / Renaissance et Réforme 30.1, Winter/hiver 2006 
104 BRENDA DUNN-LARDEAU

C'est en 1518 qu'Érasme publia ses Colloques, ce manuel scolaire de conversation latine destiné à la jeunesse européenne qui comportait saynètes et dialogues des plus vivants. Ce recueil fut souvent réédité et son auteur l'enrichit de nouveaux colloques jusqu'en 1533 , date de la dernière édition de son vivant ${ }^{3}$.

D'ailleurs, Érasme se montre très conscient d'y avoir traité d'autres choses que de la pureté de la langue latine dans Sur l'Utilité des Colloques (De Utilitate Colloquiorum), texte qu'il publia en 1526 à la suite des Colloques pour réfuter les critiques de ses détracteurs. Il argumente même comme défense du Colloque des vieillards ou le Coche: $\ll[\mathrm{N}] \mathrm{e}$ voit-on pas comme dans un miroir quelles manières d'agir il faut fuir dans la vie, et quelles autres permettent de la rendre tranquille ? Il est préférable que les jeunes gens apprennent cela par un joyeux entretien que par l'expérience $\gg^{4}$.

Certes, une attitude joyeuse est une disposition nécessaire à la pédagogie érasmienne comme en fait amplement état son De Ratione studii (1511). Outre cela, le dialogue à deux ou à plusieurs voix permet de capter l'attention des jeunes qui ont le sentiment de surprendre des conversations sur le vifs. Grâce à ce subterfuge, ils pénètrent dans les confidences de deux femmes mariées (Le Mariage ou la femme qui se plaint du mariage / Uxor mempsigamos sive conjugium), ou encore dans des lieux aussi inattendus qu'un lupanar où conversent sans complaisance un client et une prostituée (Le jeune homme et la prostituée / Adolescentis et scorti), voire au chevet de mourants (Lesfunérailles / Funus), où l'attitude de l'un, adepte de la piété traditionnelle, fait contraste à l'ars bene moriendi de l'autre, imprégné de la philosophia Christi, autre appellation, selon André Godin, de l'humanisme évangélique d'Érasme ${ }^{6}$. Ces histoires, ancrées dans les realia, n'ont pas toutes une fin heureuse, mais on s'y inquiète du sort des personnages, sort qui a des chances de s'améliorer grâce au dialogue pédagogique.

Et autour des tables animées de cinq banquets (Convivium fabulosum, varium, profanum, religiosum et sobrium), il est souvent question du bonheur selon les philosophies épicurienne, stoïcienne et chrétienne, confrontées de manière moins antagoniste que dans le De voluptate de Lorenzo Valla, tout comme de plaisirs aussi variés que la nourriture, les jardins, les lectures sacrées et profanes, sans oublier l'amitié palpable qui fuse des échanges entre les convives7. Michel Jeanneret a consacré de belles pages à la fête des mots qui rend les banquets érasmiens si plaisants. Entre autres, il a détaillé les causes des plaisirs du repas pris entre amis qui « répond aux exigences du corps, de l'esprit et de la courtoisie » :

Entre commensaux, d'un Convivium à l'autre, les thèmes, le ton, la méthode de la conversation fluctuent. Une atmosphère commune, néanmoins, surmonte les diffé- 
rences : des amis atteignent ensemble à un bien-être équilibré ou, dans un climat de sympathie humaine, le corps et l'esprit trouvent également leur compte. [...] Ils [les mangeurs] manquent rarement une occasion de souligner l'interaction du plaisir alimentaire et du plaisir verbal. Plus que jamais la bouche est à la fête, toutes activités confondues. [...] Aménager les phrases et des plats, goûter la chose et goûter le mot, ce sont deux manières de savourer pleinement les biens du monde. Les amis parlent de lièvres tandis qu'ils en mangent, débattent de vin tout en buvant $[\ldots]^{8}$

Les Colloques prodiguent même des conseils pour venir à bout des maris difficiles, voire restaurer le bonheur conjugal, en revalorisant l'acte de chair. Ainsi, dans le colloque Le mariage ou la femme qui se plaint du mariage (1523), Eulalie, heureuse en ménage, fait les recommandations suivantes à son amie, la mal mariée, Xanthippe :

Ce que j'estime devoir te recommander en tout premier lieu c'est de ne laisser éclater aucune dispute dans la chambre à coucher ou dans le lit : il faut au contraire veiller à ce qu'en ces lieux tout ne soit que fête et volupté. En effet si cet endroit, qui a été consacré pour que les offenses y soient oubliées et la bonne grâce restaurée, est profané par quelque différend ou je ne sais quelle rancœur, tout remède nous est enlevé pour le rétablissement de la tendresse mutuelle. Or il existe des femmes d'humeur si morose que, même en faisant l'amour, elles sont pleines de ressentiment et vous cherchent querelle, faisant ainsi perdre, par leur fâcheux caractère, tout l'agrément de cette volupté qui d'ordinaire chasse du cœur des hommes tout ce qu'il peut contenir d'amertume, gâtant ainsi le remède spécifique qui aurait eu le pouvoir de guérir le mari et la femme de leurs mutuelles offenses. ${ }^{9}$

Ailleurs, Érasme définit le bonheur par la négative. Par exemple, dans le colloque Sympathie (Amicitia), publié en 1533, le personnage d'Éphorin, après un long préambule de nature scientifique, emprunté à Pline, sur les affinités et inimitiés que l'on observe dans la Nature, parmi les animaux, puis, entre certains animaux et les hommes ${ }^{10}$, conclut :

Il y a des gens qui recherchent le bonheur par la magie ou l'astrologie. En ce qui me concerne, je crois que le moyen le plus sûr pour parvenir à la félicité consiste à éviter tout mode de vie à l'égard duquel on éprouve d'instinct une répugnance secrète, et à adopter celui vers lequel on se sent porté par inclination-à l'exclusion bien entendu des turpitudes. Il faut également se soustraire à la compagnie des gens dont le caractère ne convient pas au nôtre, et ne se lier qu'avec ceux pour qui ont est pris d'une affection intuitive. ${ }^{11}$

Ceci va amener ce personnage à introduire une distinction fort subtile pour réconcilier le devoir chrétien d'amour de son prochain avec la prise en compte des sympathies individuelles d'après la nature de chacun. Aussi, si l'on en croit 
106 BRENDA DUNN-LARDEAU

Éphorin : «La charité chrétienne s'étend au monde entier, mais l'intimité doit être réservée à un petit nombre $\gg^{12}$, ce qui n'est pas sans évoquer la conception élitiste de l'amitié selon les stoïciens qui aimaient l'humanité, mais non les foules.

Certes, dans les conversations des Colloques, la gaieté de ton a quelque chose d'une seconde nature entre ces gens gagnés, pour la plupart, aux bonae litterae, et même leurs disputes conservent un élégant piquant, qui sert la double finalité du placere et docere. Fait notable, quelques colloques mettent aussi en scène des marginaux, qu'Érasme ne se contente pas de laisser au ban de la société. Au contraire, il propose des pistes de solutions réalistes selon chaque état de vie, solutions, qui sont à fois de la responsabilité de l'individu et de la société. C'est le cas du colloque La Mendicité, lequel, tout en vantant avec un mélange de sérieux et d'ironie la liberté suprême dont jouit le mendiant, s'interroge sur la responsabilité des villes devant le phénomène grandissant du vagabondage plutôt que de s'en remettre uniquement aux vertus de la traditionnelle charité chrétienne individuelle. Ajoutons à cet exemple celui du colloque Le jeune homme et la prostituée (Adolescentis et scorti). Loin d'envoyer cette jeune femme faire pénitence pour le reste de ses jours dans un lieu hostile comme le font les récits hagiographiques médiévaux, le dialoguiste imagine des moyens concrets pour la sortir de son état, tel un déménagement assorti d'un emploi honorable de servante ou encore une dot remise par ce jeune homme fortuné.

Bref, Érasme se montre soucieux de revoir les rapports entre bien-être individuel et bien-être collectif, même parmi les plus humbles représentants de la société de son temps, afin que chacun accède, sinon au bonheur parfait, à tout le moins, à la dignitas hominis ou mulieris.

Outre les Colloques déjà mentionnés, véritables guides pratiques de la vie heureuse, il arrive même que certains colloques mettent en scène des religieux heureux. C'est le cas du colloque Les Franciscains qui présente sous un jour favorable deux frères mendiants qui doivent leur félicité à leur détachement proprement franciscain des biens de ce monde ${ }^{13}$. Quant au dernier colloque de 1533 L'Épicurien (Epicureus), Érasme, qui n’a cessé de critiquer le monachisme depuis les Antibarbares, sa première œuvre, fait l'éloge de ce mode de vie empreint de la tranquillité de l'épicurisme christianisé, en plus de faire un pied de nez à Luther qui l'avait traité d'épicurien ${ }^{14}$. De nombreux critiques ont relevé le paradoxe de l'épicurisme de ce chrétien. Entre autres, Franz Bierlaire a souligné, dans ce dialogue, « qui offre une image optimiste du christianisme $\gg$ l'importance de la conscience paisible et de la vraie piété qui rend heureux de telle sorte que devant ces vrais biens, il devient aisé de délaisser les biens illusoires ${ }^{15}$. De son côté, Daniel Ménager s'est interrogé à propos de cette thèse paradoxale : 
Cette thèse, Érasme peut-il la soutenir ailleurs que dans le cadre d'une fiction ? Cela n'est pas sûr. Elle ne contredit certes pas la philosophia Christi. On peut même dire qu'elle est dans l'esprit des Béatitudes. Mais dans les Colloques, et grâce aux personnages inventés, elle possède une vivacité, un pouvoir d'interrogation qu'elle n'aurait pas ailleurs. ${ }^{16}$

Ce colloque de 1533 n'annonce-t-il pas, à sa façon, l'abbaye mixte de Thélème, qui n'est envisageable qu'au sein de l'utopie rabelaisienne du Gargantua de 1534 et qui n'existe pas plus dans les realia que le monastère pouvant accueillir ce moine épicurien ? Ainsi, l'éloge de ces deux modèles de vie heureuse et pieuse se double d'une critique à peine voilée des conditions dans la société d'alors peu propices à leur réalisation.

D'autres leçons de bonheur, abordées tantôt de manière positive, tantôt de manière négative, se trouvent dans Le Colloque des vieillards ou Le Coche (Senile Colloquium) où, grâce à la copia verborum et rerum, les idées sur la félicité sont illustrées à merveille par la festivitas de la pédagogie érasmienne.

\section{Le Colloque des vieillards ou Le Coche}

Le Colloque des vieillards ou Le Coche de 1524 réunit quatre sexagénaires qui ne s'étaient pas revus depuis leurs années d'études, à Paris, quarante ans auparavant. Au cours d'un voyage en coche jusqu'à Anvers, ils feront le bilan de leur vie. Ceuxci ont tous vécu d'une manière différente. Eusèbe, après des études en médecine et en théologie, vit tranquillement d'une riche prébende ; Pampire, pour se punir d'avoir tout dilapidé au jeu de dés l'argent que son père lui avait confié, est passé de couvent en couvent avant de revenir, apaisé, à sa vocation de marchand; Polygame, vieilli et usé par sa vie de noceur, a été marié huit fois tandis que Glycion, veuf et encore resplendissant de jeunesse malgré ses 66 ans, vit heureux, persuadé que le choix d'un genre de vie qui convient aux aptitudes et aux capacités de chacun « a une influence déterminante sur notre bien-être en ce monde $\gg^{17}$.

Le Colloque des vieillards offre l'occasion de réfléchir sur les modèles des genres de vie inspirés par la tradition antique philosophique. L'originalité d'Érasme est, entre autres, de repenser les classifications traditionnelles (celle des trois vies : voluptueuse, active et contemplative) de Platon et d'Aristote pour les adapter à une société qui cherche à réconcilier honestas et voluptas, tout en conservant l'idée de juste milieu (sober mediocritas) de l'Éthique à Nicomaque ${ }^{18}$. Par ailleurs, Érasme, en bon humaniste, se démarque de l'attitude médiévale qui donnait préséance aux besoins du groupe par rapport à ceux de l'individu. La question du choix de l'état 
108 BRENDA DUNN-LARDEAU

de chacun lui tenait à cœur non seulement parce que c'était dans l'air du temps, mais aussi parce que le jeune Désiré Érasme avait souffert d'une vocation forcée contraire à son inclination personnelle.

On conviendra que la notion de choix personnel présuppose que chacun dispose d'une part de liberté. Or, une question centrale en cette année 1524 est celle du libre arbitre et du différend qui l'oppose à Luther. Ainsi, il faut rappeler que non seulement la publication de la Diatribe sur le libre arbitre de 1524 avait-elle déjà été ébauchée dans une version de février 1524, mais qu'Érasme en avait déjà développé des thèmes dans une lettre du $1^{\mathrm{er}}$ février $1523^{19}$. Par ailleurs, grâce aux travaux de F. Bierlaire, on sait qu'Érasme a ajouté quatre colloques à l'édition des Familiarum colloquiorum formulae de mars 1524, qui survient six mois avant celle du De libero arbitrio de septembre 1524. Parmi ces quatre colloques figurent Inquisitio et le Senile Colloquium. Le premier, qui n'était pas écrit pour les enfants, est considéré par la critique comme un dernier effort de conciliation avec Luther. Dans un esprit œcuménique avant la lettre, Aule (Érasme) interroge, de manière serrée, Barbat (Luther) ce qui réussit à faire ressortir leurs nombreux accords qui l'emportent sur leurs désaccords en matière de foi. Il est notable aussi que les deux personnages s'entendent pour dire que le souverain bien se trouve en Dieu ${ }^{20}$. Le second est, certes, destiné à la jeunesse, et il nous semble pouvoir y reconnaître, sous une forme pragmatique, l'empreinte de certaines idées sur le libre arbitre, tel que le conçoit Érasme.

Le Senile Colloquium offre donc une discussion franche et nuancée sur l'espace de liberté qui s'était ouvert à ces quatre vieillards au temps de leur jeunesse, discussion qui s'éloigne des Moralités médiévales tout en reflétant les débats contemporains sur la liberté de l'homme. Comment chacun a-t-il exercé sa liberté dans le choix de son genre de vie ? Et surtout, quelles conséquences ces choix ont-ils eues sur la félicité humaine de chacun ? Les cas de Glycion et de Polygame, qui ont choisi des voies de bonheur on ne peut plus contrastées, sont instructifs tant par les idées qui y sont confrontées que par la rhétorique du « joyeux entretien $\gg^{21}$ qui les anime.

Dans les propos de Glycion, il y a souvent des couleurs néo-stoïques, par exemple lorsqu' il dit avoir pris sur lui « de ne rien désirer ni haïr avec passion $\gg^{22}$, ou encore que la raison doit prévaloir sur les petites contrariétés de la vie. Par ailleurs, dans sa réponse à Eusèbe qui lui demande s'il est ou non tourmenté par la crainte de la mort, Glycion établit une distinction entre la responsabilité divine et humaine qui gouverne l'au-delà et cette vie. Ceci nous vaut d'entendre le secret de son bonheur, où la conception chrétienne l'emporte, puisqu'il prend bien soin de préciser son 
acceptation, exempte de curiosité impie, de ce que lui réserve le « ciel » plutôt que sa soumission aux lois de l'univers, idée chère aux stoïciens :

[Je ne crains pas la mort] Pas plus que je ne m'afflige du jour de ma naissance. Je sais qu' il faut mourir. M'en inquiéter sans cesse abrégerait peut-être mon existence de quelques jours, cela ne saurait en aucun cas la prolonger. Aussi je m'en remets entièrement au ciel là-dessus, ne me préoccupant que de vivre agréablement et honnêtement — l'un n'allant pas sans l'autre. ${ }^{23}$

Dans cette réplique, voilà également balayées les craintes et superstitions médiévales entourant la mort. Le seul souci de Glycion est de maintenir l'équilibre entre honestas et voluptas. Chose intéressante, c'est aussi, comme le note Franz Bierlaire, ce que dit Magdalie, dans le colloque Le père abbé et la femme instruite (Abbatis et eruditae) également publié en $1524:$ : Mais comment est-il possible de vivre agréablement sans vivre selon le bien? $? \gg^{24}$

Outre cela, les paroles de Glycion reflètent une compréhension aristotélicienne du bonheur envisagé comme le résultat d'une action et non comme un état d'esprit et encore moins comme le résultat de la chance. Ceci est frappant lorsqu' il entretient Polygame de son bonheur conjugal passé et lui explique les raisons du choix de son épouse: «Mais ne te trompe pas, je ne dois pas cette félicité seulement à la chance » même si « La plupart des gens aiment au hasard et sans discernement. Moi j’ai élu, après réflexion, une femme que je puisse chérir. Du reste je l'ai épousée moins pour le plaisir que pour m'assurer une postérité. Et je n'ai vécu avec elle que huit ans, mais qui ont été des plus doux $\gg^{25}$.

Est-ce à dire que les domaines de responsabilité divine et humaine sont complètement distincts ? Non, car, pour Glycion, le lien entre les deux réside dans sa conscience :

Et comme rien ne trouble moins le repos qu'une mauvaise conscience, je ne vais pas me coucher, si j’ai commis quelque faute, avant de m'être réconcilié avec Dieu. Car la source de la véritable tranquillité ou, pour parler en grec, de l'ataraxie, c'est la paix avec Dieu [...]. ${ }^{26}$

Ce passage décrit de manière éloquente la christianisation de l'ancienne notion d'ataraxie, la tranquillité de l'âme chez les philosophes stoïciens. Outre cela, la décision de Glycion de se réconcilier avec Dieu directement plutôt que par le moyen de la confession à un prêtre dénote son humanisme évangélique. Du reste, comme il est conscient que plusieurs sont facilement envieux de ceux qui semblent extraordinairement heureux, il a renoncé à chercher une charge plus élevée et plus glorieuse. Ce comportement face à la gloire, idée qui a fasciné sous toutes ses formes 
les esprits à la Renaissance, illustre l'idéal antique délibérément choisi de la sober mediocritas que décrit Aristote dans l'Éthique à Nicomaque 27.

Mais revenons aux plaisirs auxquels Glycion se permet de goûter. Il chérissait sa femme et est resté veuf dans l'intérêt de ses enfants, renonçant ainsi aux plaisirs charnels de la vie conjugale. À son avis, le plus grand plaisir dans la vie consiste dans le temps consacré aux études même s'il n'étudie pas plus d'une heure à la fois, partageant son temps entre l'étude et le loisir, c'est-à-dire, dans son cas, entre la lecture d'un livre et la pratique du luth ou du chant. Ceci nous ramène aux joies de la vie contemplative (ou intellectuelle) des modèles antiques ${ }^{28}$. Ainsi, Glycion mène la vie active d'un laïc et jouit avec modération des plaisirs de la vie contemplative (ou intellectuelle). Ce portrait équilibré de Glycion semble pourtant incomplet, selon Franz Bierlaire, qui note : «On s'étonnera qu'il ne parle pas de ses quatre enfants ni des petits-enfants qu'il doit avoir. Comme les autres vieillards mis en scène dans les Colloques, il semble ignorer les joies de la famille et ne pas pratiquer l'art d'être grand-père $\gg^{29}$.

Quant à Polygame, il s'est astreint à la règle d'Épicure au cours de ses nombreuses aventures amoureuses, dès ses années d'études à Paris. Cette règle a été comprise selon la notion vulgaire (qui correspond à l'hédonisme), selon laquelle cette philosophie prônerait la satisfaction de chaque désir. Polygame n'a cure de l'épicurisme original où les plaisirs naturels sont réglés selon la raison ou encore de la version christianisée pratiquée par le moine du colloque L'Épicurien. Pourtant, son goût effréné pour la vie de plaisirs lui a causé plusieurs ennuis. Forcé de quitter la maison paternelle parce qu' il y avait ramené une jeune Française enceinte, Polygame lui fit d'autres enfants ou pour reprendre son ton aussi désinvolte que grossier « le coq s'en alla avec la poule et lui fit quelques poussins $\gg^{30}$, après quoi il s'endetta, malgré l'aide secrète de sa mère. Et seule la menace d'être déshérité par son père a ralenti son comportement de jouisseur irresponsable et l'a incité à épouser les jeunes femmes qu' il se contentait d'engrosser ${ }^{31}$. En effet, ce coureur de jupons a fini par se marier huit fois et faire encore plus d'enfants, même s'il se désole de ne pouvoir avoir deux ou trois épouses à la fois « comme font les coqs avec les poules $\gg^{32}$. Les métaphores animalières pour designer sa famille et lui-même expriment sans ambiguité une vie centrée sur le corps et les bas instincts. Certes, ce personnage incarne le vice de luxure comme dans les moralités du Moyen Âge, mais aussi la notion de philautie, inspirée de l'Antiquité, notion qu'Érasme connaissait bien pour avoir consacré un de ses Adages ( $\mathrm{n}^{\mathrm{O}} 292$ Les philautes) à cet amour exagéré de soi, qui rend ceux qui en sont atteints indifférents au bien-être des autres. 
D’ailleurs, pour l'Antiquité classique, la vie voluptueuse (ou de plaisirs) avait toujours été jugée inférieure aux autres dans les modèles de vie parce que sa part de satisfactions provenait uniquement de biens matériels et corporels (ce qui explique pourquoi Platon dans sa République relègue ce modèle de vie aux membres de la société qui ne sont ni philosophes, ni guerriers). Érasme semble abonder en ce sens en traçant le portrait du libidineux Polygame qui suit sa nature et fait fi de son éducation libérale de telle sorte qu'il gagne sa vie en travaillant de ses mains. Et quoique la gouaille de Polygame, qui clame par fanfaronnade n'être « jamais resté célibataire plus de dix jours $\gg 33$, va provoquer, à coup sûr, le rire chez ses jeunes lecteurs, rien chez lui ne risque de susciter l'admiration. Au contraire, ses anciens camarades, tous plus ou moins du même âge que lui, ne cachent pas leur étonnement devant les effets dévastateurs d'une sexualité débridée et des beuveries sur son corps décrépit ${ }^{34}$. Et Pampire de conclure devant ce triste spectacle : «Glycion n'a nullement vieilli, tandis qu'on prendrait Polygame pour son grand-père $\gg 35$.

Ceux qui ont fréquenté l'œuvre d'Érasme savent que ses idées circulent librement d'un texte à l'autre : une idée qui n'est ici qu'allusion, s'incarne là dans un personnage pour se voir défendre ailleurs avec la copia d'un argumentateur. Il semble en aller de même pour l'idée d'imputer à la volonté et à la responsabilité individuelles le sort de chacun dans le Senile Colloquium et la Diatribe du libre arbitre (qu'Érasme publiera en septembre 1524 et auquel il travaillait déjà quand fut publié le Senile Colloquium). Derrière la théorisation de sa conception de la liberté de cette Diatribe, on peut reconnaître les fondements moraux des comportements antithétiques d'un Glycion et d'un Polygame :

[...] je m'appuierai sur l'autorité des Anciens qui nous ont transmis que certaines semences de bien ont été implantées dans l'esprit humain : grâce à elles, dans une certaine mesure, ils [les hommes] voient et recherchent le bien. Mais s'y trouvent ajoutés des passions plus grossières qui le sollicitent en sens contraire. On nomme donc libre arbitre cette volonté qui peut se tourner d'un côté ou de l'autre. Mais bien qu'elle soit peut-être plus portée au mal qu'au bien du fait de la permanence de notre inclination au péché, personne n'est contraint au mal à moins d'y consentir. ${ }^{36}$

\section{Le Colloque des vieillards et les moralités médiévales}

Craig R. Thompson, l'éditeur et le traducteur de la version anglaise des Colloques, a écrit du Colloque des vieillards qu'il s'agissait « d'une sorte de moralité ${ }^{37}$. Cette expression mérite quelques commentaires pour mettre en relief ce qui relie encore le dialogue érasmien au théâtre médiéval et prendre la mesure de sa nouveauté par 
112 BRENDA DUNN-LARDEAU

rapport au discours sur les deux félicités. Prenons comme repère la moralité anonyme du Xv ${ }^{\mathrm{e}}$ siècle Bien Advisé, Mal Advisé, considérée comme le modèle du genre ${ }^{38}$.

Ainsi, comme dans la plupart des moralités, il y a une condamnation sans appel du plaisir terrestre, principalement des péchés de la chair ( $g u l a)$ et de la luxure (luxuria), le tout accompagné, de surcroît, d'une carte semée de lieux allégoriques, tel celui de Franche Voulenté où Bien Advisé et Mal Advisé se séparent, le premier pour suivre le chemin du bien et le second, pour s'enfoncer dans la voie du mal, buvant et jouant aux dés.

Le seul des quatre personnages du Colloque des vieillards qui s'approche le plus des personnages allégoriques d'une moralité est Polygame, qui incarne le vice de la luxure, alors que les trois autres sont beaucoup plus complexes. Ajoutons que dans les moralités, la conclusion n'est pas seulement didactique, mais un rappel constant des finalités eschatologiques de telle sorte que Bien Advisé, Mal advisé se termine avec la condamnation àl'enfer du pécheur Mal Advisé tandis que les anges emportent au ciel le bon Bien Advisé. Par opposition, Érasme, dans Le Colloque des vieillards, concentre ses efforts sur le bonheur sur terre considéré comme la responsabilité de l'individu si bien que les portes de l'enfer n'ont pas à s'ouvrir dans une fin spectaculaire pour punir Polygame de ses excès, puisque son corps ravagé fournit la preuve suffisante que sa vie de plaisirs a déjà mené à des résultats fort évidents.

Et si Le Colloque des vieillards évoque par certains aspects une moralité, le ton sévère et la vision manichéenne du bien et du mal en moins, son contenu le rapproche aussi de l'Éloge de la Folie puisqu'il sert à distinguer, dans un contexte adapté à de jeunes lecteurs, les vrais bonheurs des faux dans différents genres de vies, sans l'envolée du sermon de la Moria sur la folie de la croix ${ }^{39}$. Surtout, ce dialogue reflète les débats contemporains, auxquels Érasme prit part, sur la liberté et la responsabilité humaines.

\section{Conclusion}

Somme toute, dans Le Colloque des vieillards, Érasme s'inscrit dans la modernité en rendant chacun à la fois libre, donc responsable, de son choix de carrière ainsi que du bonheur personnel qui s'en suit ici-bas. La rhétorique du « joyeux entretien » contribue aussi à moderniser le discours de moraliste, qui s' inspire des realia plutôt que d'abstraites allégories lourdement didactiques des moralités et qui n'insiste pas non plus sur les finalités eschatologiques comme dans la moralité du $\mathrm{Xv}^{\mathrm{e}}$ siècle Bien Advisé, Mal Advisé. 
Et, alors que d'autres auraient sombré dans la mélancolie, voire la résignation chrétienne, Glycion demeure un veuf serein. Celui-ci incarne un idéal d'humaniste chrétien laïc et jouit de la tranquillité en choisissant avec soin ses plaisirs en accord avec sa conscience. Outre cela, le bonheur tranquille, qui lui procure la félicité terrestre, peut être savouré sans culpabilité, dans la mesure où honestas et voluptas marchent main dans la main et que la philautie se tient loin afin de conserver l'équilibre entre le bien collectif et individuel. Polygame, le jouisseur invétéré, est le parfait contre-exemple de Glycion, ses plaisirs corporels et matériels passant avant toute chose malgré son éducation libérale. Ce dernier exemple tempère aussi l'idée prônée par Érasme de suivre la nature de chacun, car, comme dans le colloque Sympathie (Amicitia), cette idée exclut la turpitude. Surtout, une conception bien érasmienne du libre arbitre, qui peut être employé à bon ou à mauvais escient, soustend les récits de ces anciens compagnons (quoique ni l'idée ni le mot de grâce ne soit mentionnée).

Dans les Colloques, qui ont le bonheur pour thème central ou secondaire, Érasme s'évertue à créer un sentiment de contentement chez le jeune lecteur jusque dans les pages de Funus, véritable ars bene moriendi en toute sérénité. Ce manuel de conversation latine se double d'un guide théorique et pratique du bonheur, avec ses définitions de l'ataraxie et ses exempla de tranquillité de l'âme qui en incarnent la variante tantôt épicurienne, tantôt stoïcienne, mais toujours christianisée, car obtenue grâce à la paix avec Dieu, sans oublier les contre-exemples hauts en couleurs tout aussi éloquents. À la base de ces joyeux entretiens sur le bonheur humain, qui tentent de concilier honestas et voluptas, se trouve un véritable plaidoyer pour le respect de la nature et des inclinations de chacun dans le libre choix (mûrement réfléchi) de la carrière, du conjoint et même de ses amis. Et cette revendication du bien-être individuel n'empêche pas de prêter une oreille attentive au sort que la société réserve à un vagabond ou à une prostituée.

Selon les Colloques, on insiste sur l'importance de la philosophia Christi et de la conscience individuelle ou encore on critique les faux bonheurs et certaines valeurs traditionnellement associées au bonheur comme la gloire. Ces dialogues sont pénétrés, de surcroît, de l'esprit de l'Éthique à Nicomaque, où le bonheur, envisagé comme résultat d'une action et d'une responsabilité individuelle, réside dans le juste milieu.

Chose certaine, les Colloques semblent une parfaite illustration de la méthode pour parvenir au bonheur décrite dans le De Pueris d'Érasme (1529) :

Or le principe universel de la félicité humaine réside essentiellement en trois choses : la nature, la méthode et l'exercice. J'appelle nature, une aptitude et une disposition 
profondément implantée en nous pour ce qui est bien. Par le terme de méthode, je désigne une connaissance reposant sur des avertissements et des préceptes. Par exercice, j'entends l'usage de cette habitude que la nature a instaurée et qu'a développé la méthode. La nature a besoin de la méthode et l'exercice, s'il n'est pas dirigé par cette dernière, conduit à des erreurs et à des dangers sans nombre..$^{\circ}$

Érasme reste donc entièrement conséquent avec lui-même dans sa théorisation et sa pratique d'un enseignement du bonheur adressé à la jeunesse, qui a ses fondements dans sa confiance dans la nature humaine, soutenue par les bienfaits de l'éducation libérale.

\section{Notes}

1. Voir notre article « Prolégomènes à une histoire de la félicité », in Portraits $d u$ bonheur au Moyen Âge et à la Renaissance. Douze modèles de félicité céleste et terrestre, $\mathrm{n}^{\mathrm{o}}$ spécial de Memini, 6, 2002 (publié en 2003), p. 5-58 pour une synthèse des grands changements qui touchent les rapports qui existent entre félicité céleste et terrestre au cours du Moyen Âge et de la Renaissance. Voir aussi l'introduction de ce numéro de Renaissance et Réforme pour la place de la félicité dans plusieurs autres textes d'Érasme de telle sorte qu'on peut parler de la félicité comme d'une préoccupation qui sous-tend son œuvre. La recherche pour cet article a été menée avec l'appui du CRSH (Conseil de Recherches en Sciences Humaines du Canada) que nous remercions.

2. Érasme, Sur l'Utilité des Colloques in Érasme, Colloques, trad. Étienne Wolff (Paris : Imprimerie Nationale, 1992), t. 2 p. 405.

3. Franz Bierlaire, qui a déjà montré de manière magistrale comment les Colloques apportent une contribution significative à la réforme des études, des mœurs et de l'Église au XVI ${ }^{\mathrm{e}}$ siècle, a également établi l'histoire des éditions des Colloques et éclairé les allusions qui font écho à l'actualité ou à la vie d'Érasme, voir Franz Bierlaire, Les Colloques d'Érasme : réforme des études, réforme des mours et réforme de l'Église au XVI ${ }^{\mathrm{e}}$ siècle (Paris : Les Belles Lettres, 1978), et Érasme et ses Colloques : Le livre d'une vie. Préface de Léon-E. Halkin (Genève : Droz, 1977), 135 p.

4. Érasme, Sur l'Utilité des Colloques in Érasme, Colloques, trad. Wolff, op. cit., p. 404405 .

5. Ces conversations latines, qui s'inspirent des dialogues antiques, débutent souvent in media res, ce qui accentue le côté spontané de la rencontre à deux (congressus), ce qui est le cas de la plupart des Colloques, ou de celle à plusieurs, soit du banquet (convivium), pour cinq d'entre eux, voir Jacques Chomarat, Grammaire et rhétorique chez Érasme (Paris : Belles-Lettres, 1981), vol. 2, p. 849-930, pour la classification des Colloques. Ajoutons, toutefois, qu'il s'en faut de peu que le Colloque des Vieillards se transforme en un Banquet non seulement grâce à cette conversation franche entre 
quatre amis plutôt que deux, mais aussi parce qu'Eusèbe, l'un des compagnons, suggère de poursuivre cet entretien dans une auberge, une fois le Coche arrivé à Anvers, et, vraisemblablement, autour d'une table.

6. André Godin, rédacteur de l'entrée «Philosophia Christi » in Érasme, éd. Claude Blum, André Godin, Jean-Claude Margolin et Daniel Ménager (Paris : Robert Laffont, 1992), p. cxciv.

7. Jacques Chomarat et Daniel Ménager, éd., Érasme, Cinq banquets. Texte et traduction par B. Boudou (Paris : Vrin, 1981).

8. Michel Jeanneret, Des mets et des mots. Banquets et propos de table à la Renaissance (Paris : Librairie José Corti, 1987), p. 167-169 pour les extraits cités.

9. Érasme, op. cit., p. 287. Émile V. Telle a montré comment les Colloques matrimoniaux ainsi que la Déclamation sur le mariage contribuaient à la revalorisation du mariage chrétien, voir Emile V. Telle, Érasme de Rotterdam et le septième sacrement : étude d'évangélisme matrimonial au XVI ${ }^{\mathrm{e}}$ siècle et contribution à la biographie intellectuelle d'Érasme (Genève : Librairie Droz, 1954), voir aussi l'introduction de ce numéro de Renaissance et Réforme pour d'autres exemples des possibilités du bonheur conjugal dans l' œuvre d'Érasme.

10. F. Bierlaire, Les Colloques d'Érasme: réforme des études, réforme des mours et réforme de l'Église au $X V I^{\mathrm{e}}$ siècle, op. cit., p. 118.

11. Érasme. Colloques, trad., Wolff, op. cit., t. II, p. 362.

12. Ibid.

13. Jean-Claude Margolin soulignait que Madame Delcourt avait judicieusement remarqué que le colloque des Franciscains ne devait pas « être traduit, comme on le fait ordinairement, par Les riches Mendiants (avec une intention ironique et satirique), mais bien par les Mendiants fortunés » en raison justement de leur pauvreté selon la stricte observance franciscaine dans le chapitre « Coup d'œil humaniste et satirique sur le monde : les Colloques », in Érasme par lui-même (Paris : Seuil, 1965), p. 62 .

14. Pour une analyse détaillée de tous les replis de la pensée épicurienne dans l'œuvre d'Érasme y compris dans le colloque L'Épicurien, où la philosophie de ce moine ne doit plus rien à l'atomisme épicurien, voir l'article de Peter Bietenholz, « Felicitas (eudaimonia), ou les promenades d'Érasme dans le Jardin d'Épicure » dans ce numéro de Renaissance et Réforme.

15. Franz Bierlaire, Érasme et ses Colloques, op. cit., p. 114-115 et p. 115 pour la citation.

16. Daniel Ménager, Érasme. 1469-1536 (Paris : Desclée de Brouwer, 2003), p. 119.

17. Érasme. Colloques, trad. Wolff, op. cit., t. 1, p. 334. Rappelons que l'intérêt moral du Senile Colloquium l'épargna de la condamnation partielle des Colloques par la Sorbonne en 1526, mais non de la mise à l'Index suite au Concile de Trente qui frappa toute l'œuvre d'Érasme en 1559, voir Franz Bierlaire, Les Colloques d'Érasme : réforme des études, réforme des mœurs et réforme de l'Église au XVI ${ }^{\mathrm{e}}$ siècle, op. cit., p. 226, pour le détail des censures. Ajoutons qu'Érasme s'intéressera aussi à la question du choix de la « carrière » pour la jeune fille dans les colloques La Jeune Fille ennemie du mariage et La Jeune Fille repentante même si ces choix d'avenir sont beaucoup moins variés 
que pour le jeune homme et se limitent, en fait, à décider entre le mariage ou le couvent. Par rapport à cette tradition, Érasme innove en imaginant que le personnage de Catherine aurait aussi la possibilité de vivre une vie pieuse à la maison sans avoir à se marier contre son goût ou à aller dans un couvent aux moeurs douteuses. En effet, après avoir persuadé ses parents de la laisser aller au couvent, dont les recruteurs lui avaient brossé un tableau idyllique, elle menace par après de se suicider si sa famille refuse de venir l'en retirer, en constatant l'abîme entre son idéal et la réalité. Voir pour la contribution d'Érasme à l'éclosion d'une plus grande variété de possibilités de genre de vie pour la jeune fille de la Renaissance notre article « La place de La Comédie des quatre femmes de Marguerite de Navarre (1542) dans le discours sur le célibat volontaire comme modèle de félicité de l'Arioste à G. Suchon $\gg$, dans Renaissance and Reformation / Renaissance et Réforme 26.4 (2002), nº spécial « Quêtes spirituelles et actualités contemporaines dans le théâtre de Marguerite de Navarre », p. 113-139.

18. Robert Joly, Le thème philosophique des genres de vie dans l'antiquité classique, vol. 51, fasc. 3 (Bruxelles: Palais des Académies, 1956).

19. A. Godin, Introduction au Dialogue du libre arbitre in Érasme, op. cit., p. 686-688.

20. F. Bierlaire, Érasme et ses Colloques, op. cit., p. 70-72.

21. Les cas des deux autres compères, Pampire et Eusèbe, ont été examinés dans Brenda Dunn-Lardeau, « Erasmus' Senile Colloquium (1524) or a 'joyous conversation' on Happiness for Young People » in , Clare Bradford, and Valerie Coghlan, éd., Expectations and Experiences in Children's Literature. Proceedings of the 17th IRSCL Biennal Congress, 13-17th August 2005, Dublin, (Lichfield, U.K.: Pied Piper Publishing, 2007) p.89-98. Le premier a trouvé sa voie après un très long processus d'essais et d'erreurs de jeunesse, car sa quête de vertu et de sainteté, qui l'a mené de couvent en couvent pour se punir de ses erreurs, n'a servi qu'à lui montrer un attachement exagéré aux cérémonies et aux différences d'habits entre les congrégations plutôt qu'une piété authentique. Quant au second, qui a accepté une prébende même si la vie religieuse ne lui convient pas tout à fait, ses études de théologie et de médecine ont fait de lui un modèle de self-fashioning renaissant ; aussi, la modération qui gouverne sa vie lui vaut également la tranquillité chrétienne.

22. Érasme. Colloques, trad., Wolff, op. cit., t. I, p. 337.

23. Ibid., p. 338 .

24. F. Bierlaire, Les Colloques d'Érasme : réforme des études, réforme des mours et réforme de l'Église au $X V I^{\mathrm{e}}$ siècle, op. cit., p. 188, n. 2.

25. Érasme. Colloques, trad. Wolff, t. 1, p. 334-335.

26. Érasme. Colloques, ibid., p. 337-338.

27. Cette idée de gloire, qui se résume à deux répliques dans le Senile Colloquium (ibid, p. 335), sera reprise, en 1531, grâce aux ressources de la copia, dans le colloque L'amoureux de la gloire où l'on distingue plusieurs types de gloire, entre autres, la réputation et le souvenir de soi ou de ses œuvres qu'on laisse dans son entourage, de son vivant ou à titre posthume. Il y est aussi question des inconvénients qui accompagnent la gloire et de son refus par les stoïciens, dont la seule récompense est de pratiquer la 
vertu. Sur les divers aspects de ce thème, voir Françoise Joukovsky, La gloire dans la poésie française et néo-latine $d u X V I^{\mathrm{e}}$ siècle (des Rhétoriqueurs à Agrippa d'Aubigné) (Genève : Droz, 1969), $637 \mathrm{p}$.

28. On se souviendra que dans sa République, Platon réserve la vie contemplative aux philosophes et la vie active aux guerriers, bien qu'au cours du Moyen Âge la première fût assimilée à la vie religieuse et la seconde, identifiée à celle des laïcs.

29. F. Bierlaire, Les Colloques d'Érasme : réforme des études, réforme des mours et réforme de l'Église au $X V I^{\mathrm{e}}$ siècle, op. cit., p. 188. Sur ce personnage, F. Bierlaire note également : «Il est évidemment tentant de ne voir dans les différents modèles proposés par Érasme à ses lecteurs qu'un seul et même personnage qui vieillirait au fil des éditions. Gaspar [de Confabulatio pia] deviendrait Glycion, après avoir été Eusèbe, l'hôte du Convivium religiosum, et il mourrait dans Funus sous le nom de Corneille $\gg$ tout en relevant que cette parenté certaine du portrait du « chrétien accompli selon Érasme est beaucoup plus complexe et il ne peut être achevé que grâce aux signalements fournis dans d'autres colloques $\gg$, ibid., p. 189 pour les deux citations.

30. Érasme, Colloques, trad., Wolff, op. cit., t. I, p. 340.

31. Ce colloque semble suggérer que les valeurs morales discutables du père ont peutêtre contribué au relâchement de celles du fils. N'est-il pas étrange, par exemple, que le père ordonne à son fils de se marier, mais qu'il lui propose une fille du pays plutôt que cette Française, comme on pourrait s'y attendre ? Ceci laisse croire qu'il place les convenances au-dessus d'une conduite véritablement morale. Aussi, même si l'on n'en saura pas davantage, dans ce colloque, sur le sort des petits, on rappelle opportunément que cette jeune Française avait, en fait, abandonné son mari français plusieurs années auparavant.

32. Érasme, Colloques, trad., Wolff, op. cit., t. I, p. 341.

33. Ibid., p. 341.

34. L'insistance d'Érasme sur la nécessité d'initier très tôt la jeunesse aux choses de la vie, en théorie, comme dans le Senile Colloquium, plutôt que par l'expérience afin d'éviter de fâcheuses conséquences, trouve encore un écho à la fin du XVI $\mathrm{I}^{\mathrm{e}}$ siècle dans la critique ironique que fait Montaigne de l'éducation de son temps. Selon ce dernier, qui possédait un exemplaire des Colloquia, les écoliers avaient déjà la vérole avant d'avoir lu Aristote sur la tempérance : « On nous aprent à vivre quand la vie est passée. Cent escollers ont pris la verolle avant que d'estre arrivez à leur leçon d'Aristote, de la temperance. » : Michel de Montaigne, Essais, éd. Pierre Villey (Paris : Presses Universitaires de France, 1988 [1965]), t. 1, Essai 26, p. 163.

35. Érasme, Colloques, trad. E. Wolff, t. I, p. 333.

36. Érasme, éd. Claude Blum, André Godin, Jean-Claude Margolin et Daniel Ménager, op. cit., p. 746.

37. Erasmus, Colloquies, trad. et notes de Craig R. Thompson in Collected Works of Erasmus (Toronto, Buffalo, London: University of Toronto Press, 1977), vol. 39, p. 448, notre traduction de $\ll$ a kind of morality play $»$. 
118 BRENDA DUNN-LARDEAU

38. Au sujet de cette moralité, voir les travaux publiés par Yves Le Hir, « Quelques indications scéniques dans la moralité Bien Advisé et Mal Advisé », Bibliothèque d'Humanisme et Renaissance, 46/2 (1984), p. 399-405; Alan E. Knight, « The Condemnation of Pleasure in Late Medieval French Morality Plays », The French Review, 57/1 (1983), p. 1-9; et Jonathan Beck, « La place de la moralité de Bien Avisé Mal Avisé dans le théâtre en France au Moyen Âge », Le Théâtre français des années 1450-1550 : état actuel des recherches, éd. Olga Anna Duhl, Actes 10 (Dijon : Centre de Recherches Le Texte et l'Édition, 2002), p. 15-24.

39. Daniel Ménager a aussi rapproché ces deux œuvres en soulignant leur parenté de perspective sur le monde. Après avoir observé que l'écriture des Colloques permettait à Érasme « de prendre ses distances avec le monde, et, du même coup, d'en souffrir un peu moins », ce critique ajoute que « pour son plus grand plaisir, il transforme le monde en spectacle. Pour un peu, il en rirait comme les dieux rient de celui qu'offre le monde dans l'Éloge de la Folie (ch. 48) » dans Daniel Ménager, Érasme. 1469-1536, op. cit., p. 117.

40. Rappelons que Jean-Claude Margolin, l'éditeur du De Pueris, a noté que ce principe tripartite de la félicité humaine de ce passage était emprunté aux Epistulae ad Lucilium (Lettres à Lucilius), LXxıı de Sénèque, Érasme, op. cit., p. 497. 